\title{
Manipulative Tasks Identification by Learning and Generalizing Hand Motions
}

\author{
Diego R. Faria, Ricardo Martins, Jorge Lobo, and Jorge Dias \\ Institute of Systems and Robotics, DEEC, University of Coimbra, Portugal \\ \{diego, rmartins, jlobo, jorge\}@isr.uc.pt
}

\begin{abstract}
In this work is proposed an approach to learn patterns and recognize a manipulative task by the extracted features among multiples observations. The diversity of information such as hand motion, fingers flexure and object trajectory are important to represent a manipulative task. By using the relevant features is possible to generate a general form of the signals that represents a specific dataset of trials. The hand motion generalization process is achieved by polynomial regression. Later, given a new observation, it is performed a classification and identification of a task by using the learned features.
\end{abstract}

Keywords: Motion Patterns, Task Recognition, Task Generalization.

\section{Introduction}

An important issue for modeling and recognition of human actions and behaviors are the motion patterns found during some activity. In different daily tasks the motion assumes an important key point to describe a specific action. The variety of human activity in everyday environment is very diverse; the same way that repeated performances of the same activity by the same subject can vary, similar activities performed by different individuals are also slightly different. The basic idea behind this is: if a particular motion pattern appears many times in long-term observation, this pattern must be meaningful to a user or to a task. In this work we are focusing on manipulative tasks at trajectory level to find similarities (significant patterns) given by multiples observations. The intention is learn and generalize a specific task by the hand movement including fingers motion as well as object trajectory along the task for its recognition. This application is useful for task recognition in robot imitation learning and can be applied in the future in such way that the generalized movements can be applied to other contexts by a robot. We are not going through the imitation part, but we are focusing on the ability of learning and generalization.

\section{Contribution to Sustainability}

During the recent years, many research fields such as human-computer interface, medical rehabilitation, robotics, surveillance, sport performance analysis have focused some of their attention to the understanding and analysis of human behaviour and 
human motions. Others examples can be seen in the field of entertainment such as games that use natural user interfaces where sensors grab the human motion to interact with the game. All these research fields are searching for new solutions to improve the standard quality levels of human living conditions, generalizing the access to high quality services. Motion pattern analysis is one of the key elements to the development of those services.

The contribution of this work is an approach to learn relevant features in manipulative tasks by finding similarities among hand motions where is possible to generalize manipulative movements that can be applied to different contexts. This kind of approach can be used in the future to endow robots by imitation learning as well for recognition of a specific action to interact in a human environment to assist people in different tasks, compensating the absence of specialized human resources. Robotics can be used in medicine to assist in surgeries, rehabilitation, and also for complex task which is dangerous for human beings. These applications can contribute to the technological innovation for sustainability.

\section{Related Work}

In [2] is presented a programming by demonstration framework where relevant features of a given task are learned and then generalized for different contexts. Human demonstrator teaches manipulative tasks for a humanoid robot. Through GMM/BMM the signals are encoded to provide a spatio-temporal correlation. The trajectories are then generalized by using GMR. The authors in [3] presented an approach to find repeated motion patterns in long motion sequences. They state that if a point at a given instant of time, belongs to a set of repeated patterns, and then many similar shaped segments exist around that data point. They encode the characteristic point with partly locality sensitive hashing and find the repeated patterns using dynamic programming. In [4] is proposed a general approach to learn motor skills form human demonstrations. The authors have developed a library of movements by labeling each recorded movement according to task and context. By using Non-Linear differential equations they could learn and generalizing the movements.

\section{Proposed Approach}

Inside the neuroscience field we can find a decomposition of a typical human manipulation movement on different stages [1]. Actions phases are defined as manipulative activities involving series of primitives and events. In this work, the actions phases are used to find motion patterns in each one. In Fig.1 is possible to identify the actions phases and the events that happens among them. In each phase, it is possible to detect primitives to describe better an action. Those represented action phases are a high level segmentation of simple manipulative tasks. For the tasks that need to have in-hand manipulation (re-grasp or change the orientation of the object along the movement), the segmentation becomes more complex, the transport phase can enclose or can be changed to in-hand manipulation. Fig.2 shows the steps of the proposed approach. 


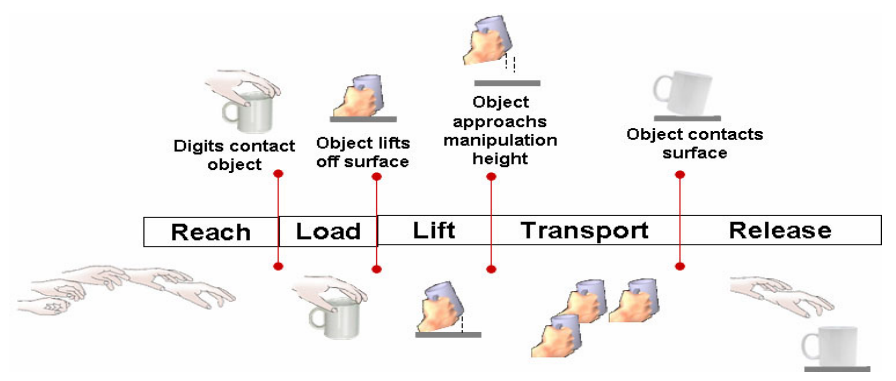

Fig. 1. Defined action phases for the manipulative tasks presented in this work

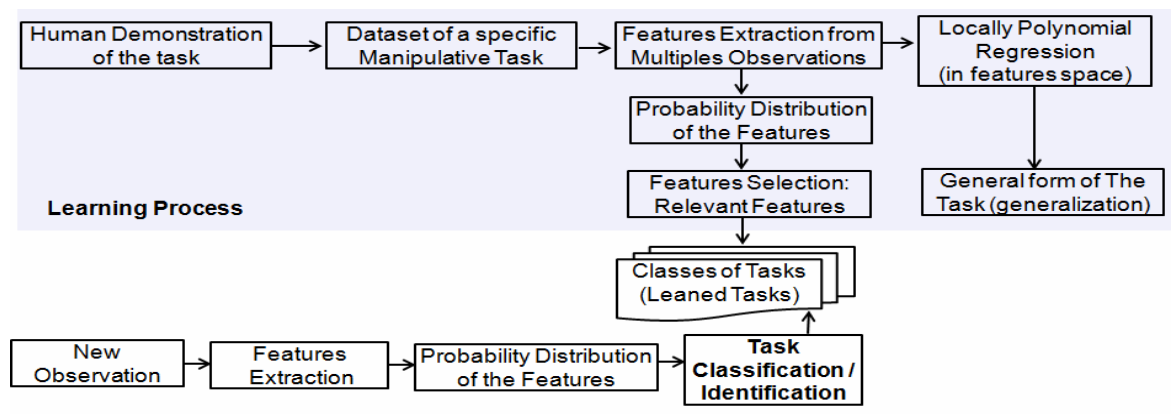

Fig. 2. Steps of the proposed approach

\subsection{Experimental Setup and Data Acquisition}

For the data acquisition is used: Polhemus magnetic motion tacking system [5]; TekScan grip [6] a tactile sensor for force feedback and CyberGlove II [7] for fingers flexure measurement. Each magnetic sensor has 6DoF (3D position and Euler angles). The magnetic sensors were attached to the fingertips to track the hand and fingers movements and also to track the object pose. The setup (Fig.3) for the experiments is
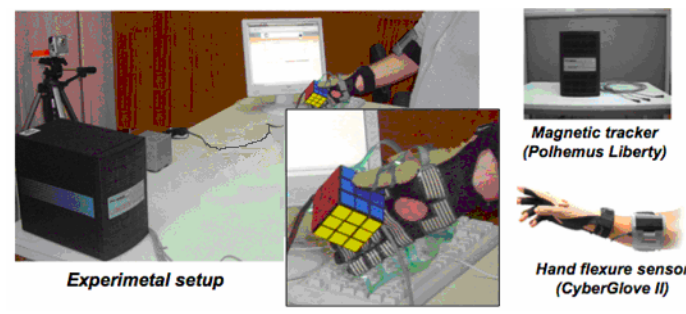
composed of a wooden table, and the experiments were executed by a subject seated in front of the table.

Fig. 3. Experimental Setup: Devices for the experiments

To facilitate the detection of each action phase by analyzing the sensors data, the sensors synchronization was needed. A distributed data acquisition was adopted, where a trigger defines the start and end of the acquisition. This way by looking to the 
data it is possible to identify some events which enable to detect the beginning and end of an action phase in a movement. Some assumption are adopted as described in previous work [8] where some rules need to be satisfied, for example, reaching: it is defined while there is hand motion, the object is static, no force pressure from tactile sensing, and variance on the fingers flexure; load phase: hand motion, force feedback, no movement of the object.

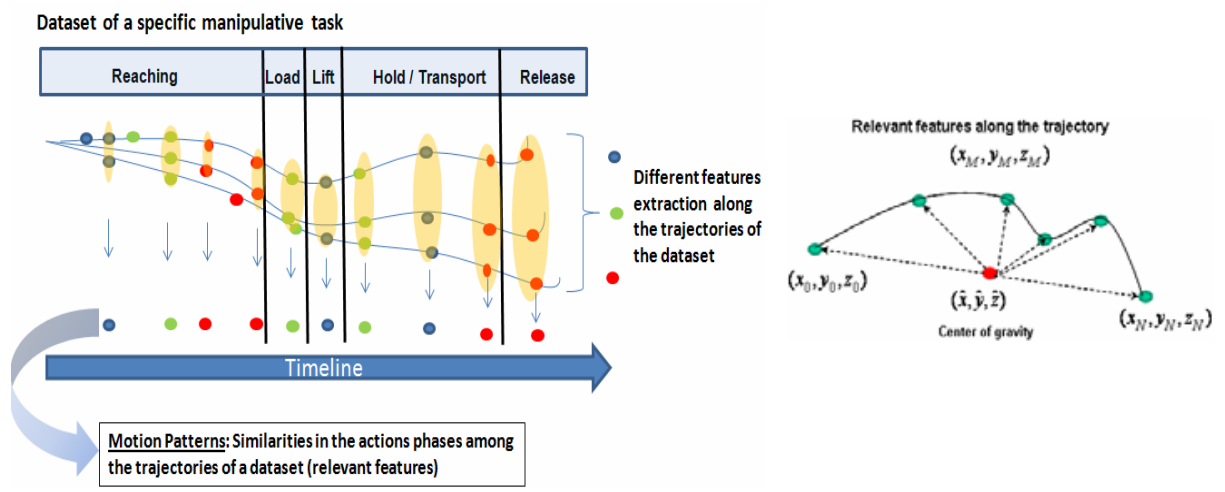

Fig. 4. - (a) Motion Patterns: Similarities detection in the action phases of the trajectories of a dataset of a manipulative task; (b) Distance of the learned features from the center of gravity

\subsection{Motion Patterns: Features Extraction and Similarities among Trajectories}

An example of the features selection is presented in Fig.4 (a). Given a dataset of hand trajectories of a task, we want to find the similarities among all trajectories, repeated patterns that are the relevant features to generate a generalized one. The idea is to detect features in each action phase of all trajectories of a dataset, then it is computed the probability distribution of these features. The algorithm selects the type of feature with higher occurrence in all trajectories by looking for the each feature coordinates, i.e. it is verified all first features in all trajectories and select the type of feature with high probability in that position and so on, for all second features, third until de last one. At the end, the features with high similarities among the trajectories are kept and by applying an interpolation among the features positions it is possible to have a general trajectory. The classes of features that is used to describe a trajectory are curvatures and hand orientation that vary during the task performance. In previous work [9] a probabilistic approach was developed for hand trajectory classification where curvatures and hand orientation where detected in 3D space. Here we are following the same idea for feature extraction.

\subsection{Patterns from Different Sensors Modality}

Other type of features is also taken into account: the fingers distances (thumb to index; index to middle and so on). The distances variance happens along to the hand trajectory, examples include hand aperture, a grasping, etc. It will also help to 
differentiate each phase of a task. At each point of the fingers trajectories is computed a mean distance of the sum of squared Euclidean distances of the fingers (1). Inside of each action phase there are many $3 \mathrm{D}$ points so that it is possible to compute $N$ distances. An alternative to represent each action phase is to compute the average of all computed mean distances (1).

$$
D_{-} a v g=\frac{1}{N} \sum_{i=1}^{N} D_{i}
$$

where

$$
\begin{gathered}
D=\sum_{k=1}^{N}\left(d_{k}\right)^{2} \\
d_{i j}=\sqrt{\left(x_{i}-x_{j}\right)^{2}+\left(y_{i}-y_{j}\right)^{2}+\left(z_{i}-z_{j}\right)^{2}} .
\end{gathered}
$$

The signals of the cyber-glove represent degrees of flexure (e.g. 0-255). An easy way to use this information is defining some grasp types such as cylindrical grasping, spherical grasping, in different levels of flexure and also defining some type of gestures, i.e. extension and flexion of the hand in different degrees of flexure (e.g. low, medium, high). After a learning stage by analyzing many observations of the same gesture it is possible to know the degree of flexure for each finger for each grasp type. In each task, it is necessary to identify the types of the defined grasping/gesture and then compute the probability distribution P(Grasp I Observation) of each one along the action phases of the task.

\subsection{Task Representation by General Form of the Trajectories}

The representation of a dataset of a specific task at trajectory level is given by the general form of the data which is achieved after selecting the relevant features and then applying a regression on the data to generalize it. The spatio-temporal information is used to apply a polynomial regression to fit the data to have a smoothed trajectory of the manipulative task. The polynomial regression was chosen due to the curvilinear response during the fit and it can be adjusted because it is a special case of multiple linear regressions model. We are adopting the quadratic form of the model, a polynomial regression of second order. Although polynomial regression fits a nonlinear model to the data, as a statistical estimation problem, it is linear, in the sense that the regression function is linear in the unknown parameters that are estimated from the data. The general model of second order polynomial regression is given by:

$$
Y_{i}=\beta_{0}+\beta_{1} x_{i}+\beta_{11} x_{i}^{2}+\varepsilon_{i}
$$

where $x_{i}=X_{i}-\bar{X}$ and $\varepsilon$ is an unobserved random error with mean zero conditioned on a scalar variable; $\varepsilon$ can be computed as error of least square fitting; $\beta$ minimizes the least square error.

In our case, due to the type of trajectories, to fit correctly the curves, the regression need to be done locally, at some parts of the trajectory, Example of regression in subregions of the trajectories is shown in Fig.5. 


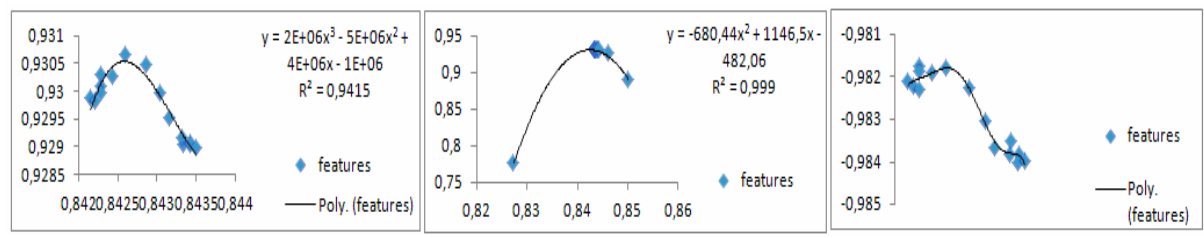

Fig. 5. - Regression applied on sub-regions of an action phase of a manipulative task. 2D view: left and middle images: $x, y$ view; right image: $x, z$.

\subsection{Task Identification}

A new task can be recognized by matching a prototype (learned task) to a new observation or via classification where there many task classes. In the case of the hand trajectory, some properties of the learned features (translation invariance) as shown in Fig.4 (b) can be used also in the matching. The learned features is used for the matching between a prototype (generalized information) to a new observation.

Another alternative is applying continuous classification based on multiplicative updates of beliefs by Bayesian technique (5) taking in consideration the learned observations (relevant features of the general form of signals). First it is identified if the task to be classified has all action phases of the learned task, and then it is possible to classify it.

$$
P\left(G_{k+1} \mid c_{k+1}, i\right)=\frac{P\left(c_{k+1} \mid G, i\right) P\left(c \_o b j_{k+1} \mid G, i\right) P\left(o_{k+1} \mid G, i\right) P\left(h_{k+1} \mid G, i\right) P(G)}{\sum_{j} P\left(g_{j} \mid c_{k+1}, c_{-} o b j_{k+1}, o_{k+1}, h_{k+1}, i\right)} .
$$

To understand the general classification model some definitions are done: $g$ is a known task goal from all possible $G ; c$ is a certain value of feature $C$ (Curvature types) found in the hand trajectories; $C_{-} \_$obj: curvatures found in the object trajectories; $H$ : the grasping type learned from the data-glove signals; $o$ : a certain value of feature $O$ (hand orientation types) $i$ is a given index from all possible action phases $A$. For more details about a methodology for some features extraction and their probability distribution see [9]. The probability $P(c \mid g i)$ that a feature $C$ has certain value $c$ can be defined by learning the probability distribution $P(C \mid G A) ; P(o \mid g i)$ of feature $O$ learning $P(O \mid G A) ; P(h \mid g i)$ learning $P(H \mid G A)$ and $P\left(c \_o b j \mid g i\right)$ of feature $C \_o b j$ learning $P\left(C \_o b j \mid G A\right)$.

\section{Results}

The trajectories that were used are pick-up and place and pick-up and lift. In Fig.6 (a) is shown the raw data of the used dataset correspondent to the task pick-up and place (hand trajectories); (b) shows the detected action phases using the sensors information. Fig.7 (a) shows an example of the 3D positions of the features extracted (curvatures: trajectory directions) from all observations before finding similarities; (b) relevant features selection by analyzing the probability distribution of the features to know which type of feature is more relevant, later after computing the least square among all features points of the trajectories dataset we can estimate the coordinates of them; (c) example of interpolation of the features points as a function of arc length along a space curve. 

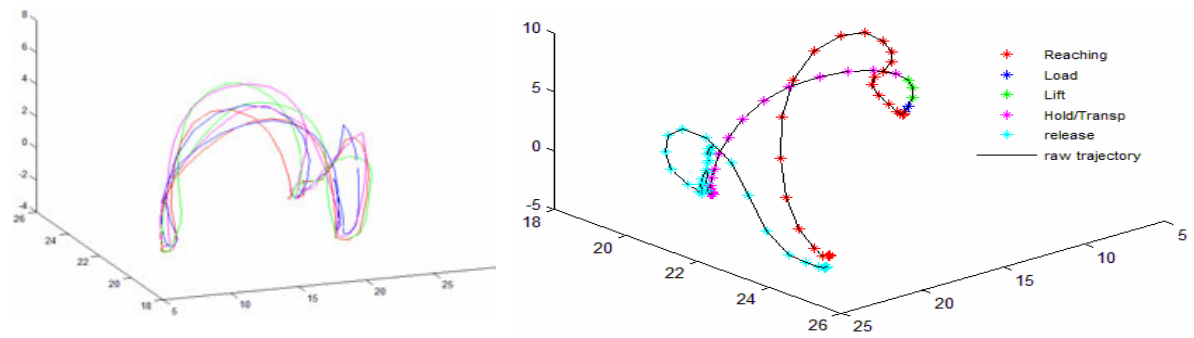

Fig. 6. - Left: Raw data(in inches): trajectories dataset (object displacement); Right: Trajectory segmentation by action phase
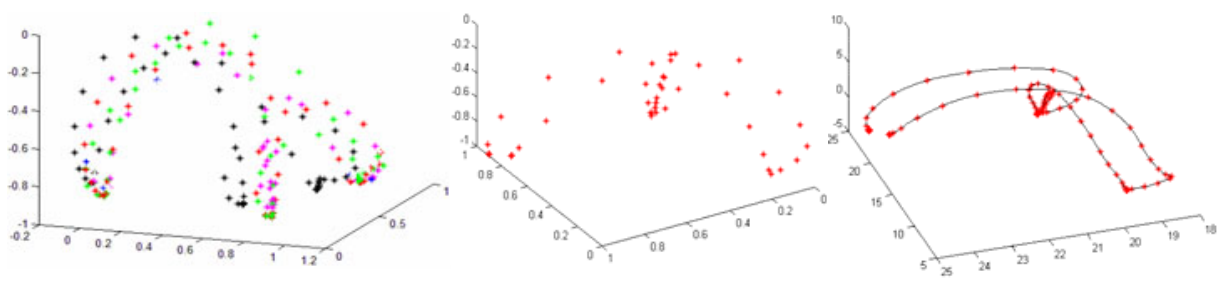

Fig. 7. - (a) Extracted Features; (b) Relevant Features (similarities among all trajectories); (c) Generalized Trajectory
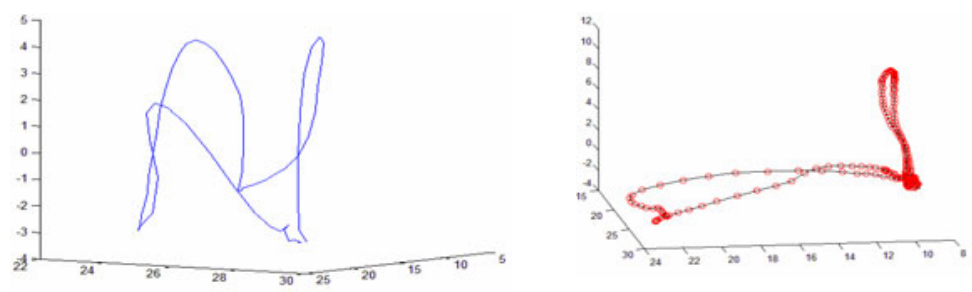

Fig. 8. - (a) New observation: trajectory to be classified (pick-up and place); (b) Trajectory of dataset pick-up and lift

Table 1. Classification Result

\begin{tabular}{lcc}
\hline \hline $\begin{array}{c}\text { Action } \\
\text { Phases }\end{array}$ & $\begin{array}{c}\text { Pick-up and place } \\
\%\end{array}$ & $\begin{array}{c}\text { Pick-up and lift } \\
\%\end{array}$ \\
\hline \hline Reaching & 45.00 & 55.00 \\
Load & 48.10 & 51.90 \\
Lift & 59.32 & 40.68 \\
Transport & 69.83 & 30.17 \\
Release & $\mathbf{7 8 . 0 0}$ & 22.00
\end{tabular}

The $2^{\text {nd }}$ and $3^{\text {rd }}$ columns show the $\%$ of the new observation belonging to pick-up and place or pick-up and lift task in each instant (part of the task). We have detected the relevant features in each phase using their probabilities to classify the new observation. The new trajectory (Fig. 8(a)) is classified as pick-up and place correctly with $78 \%$. 
The actions phases for both dataset happen in different period. Given a new trajectory we want to recognize what kind of task is it. The classification variables are updated in each action phase and at the end the variables keep the final result of the classification. Table 1 shows the result of the classification of a new observation of pick-up and place. For that, just the learned curvatures features was used in the classification model (5), in the future we intend to implement the complete model to reach better results.

\section{Conclusion and Future Work}

In this work is proposed an approach to represent and recognize a manipulative task by multiple observations performed by a human demonstrator. By finding the relevant features of a task dataset is possible to find a general form of representing a task and also recognize it. The preliminary results motivate us to follow this proposed methodology to reach satisfactory results. In future work will be tested and evaluated the proposed approach by applying it in different trials of different manipulative tasks and different sensors signals will be used.

Acknowledgments. This work is partially supported by the European project HANDLE within the $7^{\circ}$ framework FP7. Diego Faria and Ricardo Martins are supported by the Portuguese Foundation for Science and Technology (FCT).

\section{References}

1. Johansson, R.S., Flanagan, J.R.: Coding and use of tactile signals from the fingertips in object manipulation tasks. Nat. Rev. Neurosci. 10, 345-359 (2009)

2. Calinon, S., Guenter, F., Billard, A.: On Learning, Representing and Generalizing a Task in a Humanoid Robot. IEEE Transactions on Systems, Man and Cybernetics, Part B 37(2), 286-298; Special issue on robot learning by observation

3. Ogawara, K., Tanabe, Y., Kurazume, R., Hasegawa, T.: Detecting repeated motion patterns via dynamic programming using motion density. In: Proc. 2009 IEEE Int. Conf. on Robotics and Automation (ICRA 2009), pp. 1743-1749 (2009)

4. Pastor, P., Hoffmann, H., Asfour, T., Schaal, S.: Learning and generalization of motor skills by learning from demonstration. In: Int. Conference on Robotics and Automation, ICRA 2009 (2009)

5. Polhemus Liberty 240/8 Motion Tracking System, http: / /www . polhemus. com/?page=Motion_Liberty

6. TekScan Grip Sensor, http: / / www.tekscan.com/medical/system-grip.html

7. CyberGlove II, http: / / www . cyberglovesystems . com

8. Faria, D.R., Martins, R., Dias, J.: Learning Motion Patterns from Multiple Observations along the Actions Phases of Manipulative Tasks. In: Workshop on Grasping Planning and Task Learning by Imitation: IEEE/RSJ IROS 2010, Taipei, Taiwan (October 2010)

9. Faria, D.R., Dias, J.: 3D Hand Trajectory Segmentation by Curvatures and Hand Orientation for Classification through a Probabilistic Approach. In: Proceedings of The IEEE/RSJ Int.Conf. on Intelligent Robots and Systems, IROS 2009, St. Louis, MO, USA (2009) 